\title{
Actitud del facilitador hacia su función de investigación
}

\author{
Facilitator's attitude towards their research function
}

\author{
Ana Julieta Guillén Márquez \\ ana_guillen08@hotmail.com \\ Código ORCID: 0000-0002-2270-0054
}

Universidad Nacional Experimental Simón Rodríguez, Venezuela

\author{
Carmen Beatriz Soto de Vilani \\ cbsotovilani@gmail.com \\ Código ORCID: 0000-0003-3383-7264 \\ Universidad Nacional Experimental \\ Simón Rodríguez, Venezuela
}

Artículo recibido en octubre 2019 | Arbitrado en noviembre 2019 | Publicado en enero 2020

\begin{abstract}
Resumen
Esta investigación tuvo como intencionalidad comprender la actitud del facilitador hacia su función de investigación en la Universidad Nacional Experimental "Simón Rodríguez" núcleo San Carlos, Estado CojedesVenezuela. En lo metodológico, se soportó en el enfoque cualitativo, paradigma interpretativo, con el método fenomenológico-hermenéutico. Desde el punto de vista de los sujetos, se entrevistó a siete informantes claves y la descripción de la realidad se realizó con la observación, apoyado de un diario de campo. La indagación arrojó que existe una debilidad en cuanto a la actitud del facilitador hacia su función docente y se deben fortalecer las políticas institucionales hacia la investigación, a través del estímulo con buena asignación presupuestaria. Parte de los descubrimientos lleva a afinar en cuanto a la situación problemática, pues existen fallas en la implementación de las políticas, en el seguimiento y control de los proyectos, además del poco seguimiento al cumplimiento de las funciones docentes.
\end{abstract}

\footnotetext{
Abstract

The purpose of this research was to understand the facilitator's attitude towards his research function at the National Experimental University "Simon Rodríguez" nucleus San Carlos, Cojedes State- Venezuela. Methodologically, it was supported in the qualitative approach, interpretive paradigm, with the phenomenological-hermeneutical method. From the point of view of the subjects, seven key informants were interviewed and the description of reality was made with observation, supported by a field diary. The investigation showed that there is a weakness in the facilitator's attitude towards his teaching role and institutional policies towards research should be strengthened, through stimulation with a good budget allocation. Part of the discoveries leads to refinement regarding the problematic situation, since there are flaws in the implementation of policies, in the monitoring and control of projects, in addition to poor monitoring of the fulfillment of teaching functions.
}

Palabras clave:

Actitud; investigación; función investigación

\section{Keywords:}

Attitude; research; research function 


\section{INTRODUCCIÓN}

Es una realidad plenamente comprobada que la investigación educativa es un bastión importante tanto en la producción de conocimientos, como para formación docente. Sin embargo, requiere de profesionales comprometidos a la par de los cambios acelerados, producto de la globalización y dispuestos a sacar provecho de la variedad de paradigmas existentes. Dicha investigación es el mecanismo idóneo pues transforma realidades y/o ayuda en la resolución de las distintas problemáticas planteadas especialmente en el ámbito universitario.

Por tanto, efectivamente, investigación y docencia deben estar relacionadas, así del acompañamiento de la extensión, formación y gestión, todos los cuales son elementos significativos íntimamente acordes a las funciones referentes al trabajo docente. En consecuencia, docencia y formación del educador deben tener atención relevante en las universidades por lo cual es necesario adaptarse a los nuevos cambios en la búsqueda del escenario que posibilite la construcción de los vínculos facilitadoruniversidad y así potenciar una cultura de investigación y formación.

En Venezuela, en ese sentido, se plantea con urgencia a juicio de Martínez (2008):

La necesidad de desafiar al docente universitario para que reconozca la importancia y la complejidad de la práctica docente que desarrolla específicamente en este nivel y estimularlo para que -en el marco de su desarrollo profesional participe en programas de formación y actualización que respondan tanto a sus necesidades individuales como a las que plantea la propia institución universitaria en los ámbitos pedagógico, profesional, personal y social. No se trata de "pedagogizar" al docente en detrimento de la necesaria profundización del conocimiento disciplinario, se trata de insistir en la necesidad de un docente cualificado que no sólo domine los contenidos disciplinares, sino que sepa enseñarlos. (p.55)

De allí, por tanto, surge lo interesante de la formación profesional del docente en su trabajo integral, pues debe estar también comprometido con la investigación por ser un proceso encaminado a la obtención de nuevos conocimientos y su aplicación para la solución de los problemas o interrogantes a nivel académico y sociales. Este aspecto indiscutiblemente será el motor del docente; pero si éste se encuentra altamente motivado; en otras palabras, si posee una actitud marcada en su dimensión afectiva y cognitiva sin problemas, donde pueda relacionarse; de manifestar sus sentimientos, emociones, conocer, conocerse y transformar la realidad.

En función de lo antes expuesto, Myers (citado por Aldana, 2011) la actitud se define:

Estados psicológicos internos que se manifiestan a través de una serie de respuestas observables, que se pueden agrupar en tres grandes dimensiones o categorías: afectivas (sentimientos evaluativos y preferencias), cognoscitivas (opiniones y creencias) y conativas o conductuales (acciones manifiestas, intenciones o tendencias a la acción). (s.n.)

La cita indicada da gran notoriedad por una parte a los aspectos inherentes a la persona, como son sus extensiones física o biológica, cognitiva, comunicativa, ética, 
estética, afectiva, social y espiritual los mismos determinan la inclinación al tema investigativo; y por otra, capacidades mentales; es decir, el conocimiento previo sobre el área, o en su defecto posibilidad que tiene el docente de aprehender conceptualmente la realidad para poder transformarla.

Aunado a la reflexión, es importante sumar lo expresado por Boffelli (2015):

La crítica y creatividad deben estar implícitas dentro del proceso de investigación ya que el mismo no es un proceso lineal, sino que está lleno de dudas y vacíos, de incertidumbres, en donde son necesarias la crítica y la creatividad para seguir adelante. (p.12)

La autora del presente estudio da realce a este punto, por formar parte del proceso hacia la producción de conocimiento, donde se suma la capacidad de aprehender al problema de la investigación, su interpretación y el acercamiento de los contenidos epistemológicos. En consecuencia, a pesar de estos factores relevantes, las universidades venezolanas presentan un conjunto de dificultades sobre el tema de investigación abordado en el presente trabajo. Por ello, la crítica debe formar parte de la evaluación, que lleve a la toma de decisiones acertadas para enfrentar la situación.

Sobre el particular es necesario resaltar la problemática de las instituciones universitarias, en especial con respecto a su cultura investigativa pobre, motivado posiblemente a factores de orden económico y peor aún, la establecida de exigir a los participantes o estudiantes investigar; siendo muy pocos los docentes investigadores. Castro (2015, p. 10 citado por Boffelli) indica:

Los trabajos de investigación que se realizan en nuestras universidades, el motivo académico que las conduce, las tendencias de resolver los problemas de investigación sobre las mismas y con las mismas orientaciones que enseñaron y aprendieron, la escasa preparación y motivación para emprender la tarea de investigar, la formación investigativa enmarcada dentro del método científico clásico positivista, la formación en la investigación limitada al aula de clase con el fin único de demostrar competencias en el manejo de herramientas investigativas, la enseñanza de la investigación bajo las orientaciones de docentes que no investigan, centradas en un proceso informacional de transmisión de un profesor emisor a un alumno receptor. (p.8)

Desde esta perspectiva, el estado Cojedes, no escapa a la realidad abordada, asiento de la Universidad Nacional Experimental "Simón Rodríguez" (UNESR) Núcleo San Carlos, donde la situación observada por las investigadoras en su experiencia docente visualiza el incumplimiento del reglamento para el personal docente y de investigación de la UNESR, en su artículo o l "el personal (...) de investigación (...) está integrado por quienes cumplen funciones de docencia, investigación, interacción comunitaria" (s.n.). Esta condición obliga a todos los docentes a participar en actividades de investigación y divulgación, incentivando en los participantes el desarrollo de proyectos orientados a solucionar problemáticas institucionales y/o sociales. 
Otra función fundamental de la Universidad, es la Ley de Universidades (1970) artículo № 3:

Las Universidades deben realizar una función rectora en la educación, la cultura y la ciencia. Para cumplir esta misión sus funciones se dirigirán a crear, asimilar y difundir el saber mediante la investigación y la enseñanza... y formar los equipos profesionales y técnicos que necesita la Nación para su desarrollo y progreso. (p.l)

En ese mismo orden de ideas, importante el Reglamento General de Educación Avanzada de la Universidad
Nacional Experimental "Simón Rodríguez", el cual establece un cúmulo de objetivos relacionados con la investigación propiciando el estímulo de la creación intelectual en los diferentes campos del saber científico asumiendo la investigación como función central.

A pesar de lo anterior los síntomas del problema evidencian que son muy pocos los facilitadores dedicados a la investigación, esto según lo reflejado en las estadísticas proporcionadas por la Subdirección de Educación, mostrándose un total de ochenta y dos (82) docentes activos dentro de la universidad, desglosados según la tabla abajo descrita, solo el 46\% está trabajando en proyectos de investigación.

Tabla 1. Data de Facilitadores UNESR

\begin{tabular}{lccc}
\hline Descripción & Cantidad & $\begin{array}{l}\text { Facilitadores } \\
\text { Investigando }\end{array}$ & Porcentaje \\
\hline Facilitadores dedicación exclusiva & 24 & 18 & 21,95 \\
Facilitadores tiempo completo & 14 & 9 & 10,98 \\
Facilitadores medio tiempo & 9 & 5 & 6,10 \\
Facilitadores tiempo convencional & 32 & 6 & 7,32 \\
Total & $\mathbf{8 2}$ & $\mathbf{3 8}$ & $\mathbf{4 6 \%}$ \\
\hline
\end{tabular}

Fuente: Guillén (2018)

Por otra parte, la UNESR San Carlos fue fundada hace veintiún años, pero, en la actualidad no posee programas de estudios de cuarto y quinto nivel, motivado a los obstáculos de tipo burocráticos, pues depende del rectorado ubicado en la capital de la República. No obstante, en la mayoría de los núcleos de la UNESR poseen programas de postgrado.

Otro aspecto resaltante del problema, es la desmotivación de los facilitadores hacia la responsabilidad como tutores, revisores y jurados, formación e integración a las seis líneas de investigación existentes, perdiéndose la oportunidad de implicarse en los retos académicos y cambios sociales, colocándose obstáculos y comportamientos de criticar más no a aportar ideas y soluciones, a pesar de que el Reglamento General de Educación Avanzada UNESR (ob. cit) así lo establece en su Artículo 191:

b) Responsabilidades de investigación, referidas a la dedicación del profesor o profesora en actividades como: labores de investigación en la respectiva área de conocimiento. Estas actividades deberán responder al desarrollo de Proyectos de Investigación adscritos a líneas y 
programas Institucionales de investigación, que sustentan los programas de formación... (p.48)

En ese mismo orden de ideas, en el Núcleo es nula la ejecución de un programa de evaluación hacia los facilitadores y de los programas de investigación, falta de concientización acerca de su participación en las líneas de investigación, visualizándose en lo conductual un facilitador inseguro, angustiado, a no asumir responsabilidades como se describió en líneas anteriores por el temor y el conformismo a solo dedicarse a las actividades rutinarias. Asimismo, debilidad en la cultura organizacional con respecto a lo señalado en el Reglamento general de educación avanzada UNESR (ob. cit) artículo 199:

Tiene como finalidad promover, planificar, programar, desarrollar y evaluar la ejecución de Programas de formación avanzada en vinculación directa con los procesos de investigación y de interacción con las comunidades, para contribuir con el desarrollo científico, tecnológico, humanístico, social, educativo y cultural del país. (p.5l).

Por lo antes expuesto, se formulan las siguientes intencionalidades: Develar los afectos, cogniciones y la conducta de los facilitadores hacia la función de investigación en la Universidad Nacional Experimental "Simón Rodríguez" Núcleo San Carlos, estado Cojedes; describir las funciones de los facilitadores en cuanto a su rol de investigadores; y conocer los programas de formación académica institucional para la investigación que ofrece a sus facilitadores para llegar a comprender la actitud del facilitador hacia su función de investigación.

Se logró evidenciar que las funciones exigidas al facilitador no son cumplidas en su totalidad, la investigación está fuera de las tareas de docencia, extensión, gestión y formación, al igual en otras instituciones universitarias se exige al estudiante investigar; pero muchos docentes no desarrollan esa función.

Como se destacó en líneas anteriores, el tema a abordar es de gran importancia, en primer lugar, se permitió conocer las actividades del facilitador y su evaluación a nivel de formación y conductual con respecto al ejercicio de su profesión. Por otra parte, se estudió un factor relevante generador no solo de teorías, sino el tema permitirá a las universidades nacionales o internacionales ampliar la producción intelectual, aumentar el debate sobre el saber y generar proyectos de avance o desarrollo a cualquier país, en otras palabras, que la investigación sea vista como una herramienta valiosa para solucionar los grandes problemas del mundo, y con ello el desarrollo profesional del facilitador debe ser el pilar fundamental.

El estudio posibilitó el realce del papel de la formación profesional docente afectado por agentes de diferentes índoles (actitudes), al contrarrestar las debilidades en cuanto a los planes de formación e incentivar las líneas de investigación o fortalecer las existentes, mejorar el sistema retribuido, el clima laboral. En suma, se mejoraran las competencias y los intereses del facilitador.

Dentro de los aportes, se beneficiaría de manera directa a la UNESR núcleo San Carlos al contribuirle a mejorar el prestigio de la universidad, según el ranking elaborado por el Consejo Superior de 
Investigaciones Científicas (CSIC) (2012), se encuentra entre los últimos lugares y de manera indirecta al incentivar al conjunto de los facilitadores el despertar la inquietud hacia la investigación, líneas existentes y su formación con respecto al área.

Desde el punto de vista legal, se justifica la investigación al existir un marco de leyes que le darán soporte, como es la Constitución de la República, la Ley de universidades y el reglamento interno de la UNESR. Además, los distintos planes para el desarrollo de la nación desde su creación hasta el más actual, destacan el papel de todas las instituciones de educación universitaria.

Finalmente, la investigación contribuirá en despertar en los facilitadores el interés por la formación integral, a desarrollar sus potencialidades y capacidades en el orden cognoscitivo, intelectual y lógico, incluyendo el aspecto de los afectos, sentimientos y preferencias que le ayuden a convertir la enseñanza en una acción agradable y eficiente.

\section{MÉTODO}

La naturaleza de la investigación se sustenta en el enfoque cualitativo, paradigma interpretativo. El método usado fue fenomenológico -hermenéutico. Con respecto a lo cualitativo Martínez (2006) señala:

Cualidad y calidad vienen del mismo término latino qualitas, y éste deriva de qualis (cuál, qué). De modo que a la pregunta por la naturaleza o esencia de un ser: ¿qué es?, ¿cómo es?, se da la respuesta señalando o describiendo su conjunto de cualidades o la calidad del mismo. (s.n.)

Entrando en el método, Heidegger (2003) indica "el sentido metódico de la investigación de la descripción fenomenológica es una interpretación (...) La fenomenología del Dasein es hermenéutica en la importancia originaria de la palabra, significación en la que designa el quehacer de la interpretación" (p. 37). Lógicamente de acuerdo a las observaciones realizadas y de acuerdo a los resultados, se interpretó la realidad para derivar un cuerpo de consideraciones finales respecto al tema de estudio.

Los sujetos de estudio, por sus vivencias y experiencias, ayudaron a los investigadores a proveer de toda la información necesaria para realizar el estudio.

Para recolectar la información se empleó la observación, cabe destacar que la misma fue de tipo participante natural. Para este tipo de técnica se utilizó instrumento de recogida el diario de campo definido por Hurtado (2000) "una lista de indicios o aspectos relacionados con el evento de la investigación, que se pueden presentar durante la observación" (p.453). En ese sentido, la enumeración del evento a evaluar contribuyó a inventariar los elementos que fundamentarían la investigación, de allí analizar la información y generar las consideraciones generales.

Retomando el aspecto de las técnicas, se apoyó también en otro tipo, la entrevista. En el caso del presente estudio se entrevistó a siete (7) informantes claves facilitadores de la Universidad Nacional Experimental "Simón Rodríguez".

Protagonistas conocedores de la realidad porque el tema incumbe a parte de su función. La técnica antes mencionada, utiliza de instrumento el guion de entrevista semiestructurada, que contiene los datos generales de identificación del entrevistado y datos concernientes al tema de la investigación. 
La fiabilidad fue relevante porque permitió a otras personas valorar si la investigación cumple los parámetros de credibilidad y auditabilidad. Ahora bien, para reforzar este aspecto en los estudios cualitativos, importante la opinión de Guba y Lincoln (1981, citados por Castillo y Vásquez, 2003):

Hallazgos (...) pueden ser
reconocidos por los actores sociales
involucrados en la realidad como
una verdadera aproximación a lo
que ellos sienten y piensan. Lo que
implicará que se les consulte con
frecuencia durante el proceso de
investigación sobre la base de lo que
se irá sistematizando en notas de
campo con respecto a las acciones
e interacciones generadas. La
auditabilidad o conformabilidad
consiste en que otros investigadores
puedan seguir la pista al estudio;
esto se cumple debido a que se
mantiene un registro y
documentación de las ideas que
van surgiendo a lo largo del estudio.
Se hacen vídeograbaciones, y se
llevan registros escritos de las
acciones generadas. Por último, el
criterio de transferibilidad o
aplicabilidad, que se refiere a la
posibilidad de extender los
resultados del estudio a otro
contexto, por lo que es necesario
describir densamente la realidad, el
lugar y las características de las
personas envueltos en la misma.
(p.ll)

Lo antes expuesto, exigió a los investigadores estar atentos a ejecutar bien cada una de las fases del estudio de acuerdo a la planificación previa. Desde el diagnóstico y descripción de la realidad, la forma de abordaje, interacción con el ser y especificar con claridad el método, técnicas e instrumentos de recogida de la información.

\section{RESULTADOS}

Corresponde en este momento analizar la información obtenida producto de la interacción y conversación con los informantes claves quienes se les codificó con letras para una mejor identificación (A, B, C, D, E, F, G). Mediante la descripción e interpretación se abordó la nueva realidad emergente, examinando cada una de las categorías relacionadas a las unidads temáticas.

Con respecto a la motivación investigativa, esta es el impulso que mueve al investigador a interesarse a indagar sobre cualquier tema o problema, con el fin de generar nuevos conocimientos. Para Ruiz (2010):

... se concibe como el interés
manifiesto del ser, en el hacer
investigativo, este rasgo viene a
configurar un perfil observable
de las acciones, actitudes,
niveles de compromiso y
participación que el sujeto
investigador demuestra en
términos de adhesión, arraigo e
identidad (p.194)

Empezando las entrevistas, se les preguntó a los actores sociales si están realizando actualmente algún tipo de investigación y los que les motiva investigar. La informante A se encuentra investigando de manera activa, tal como se refleja en su respuesta: "Bueno sí, (...) estoy haciendo la Maestría en docencia universitaria y estoy haciendo o desarrollando una investigación cualitativa etnográfica. Para ella existe un impulso o curiosidad de descubrir: "porque existe la inquietud o la necesidad, vamos a decirlo así, de 
saber (...) del por qué estos estudiantes no tienen una prosecución académica para terminar su proyecto académico, pues su proyecto de vida académico que una veziniciaron".

Dentro del mismo relato es importante la opinión del informante B quien coincide con el primer entrevistado quien también está investigando activamente por actualización académica así lo indica: "Sí, por supuesto, estoy realizando un trabajo para mi tesis doctoral enfocada en el área de gerencia y siempre he estado permanentemente o constantemente involucrado en esa temática de investigación siempre estoy actualizándome en eso". El impulso que la mueve es su parte de crecimiento académico y no por interés relacionado a sus funciones, tal como lo refleja "por mi trabajo, ha sido por superarme, ya sea con una maestría o especialización"

En ese mismo orden de ideas, la informante $\mathrm{C}$ se mantiene activa dentro del campo científico, indicó: "Sí, (...) por supuesto desde la unidad de mis conocimientos, pues tratar de disipar un poco las dudas. (...). Su interés le mueve a conocer cosas nuevas: "en primera instancia, es porque partimos de algo que fuese novedoso".

Seguidamente el informante D, revela estar inactivo al señalar de manera tajante: "en estos momentos No, había hecho y realizado unas investigaciones por ahí en una parte de Bocatoma, donde se llama Solano, hice una tesis de grado sobre la conservación de las aéreas verdes de esa zona, es una investigación de ya hace dos años". No mostró claridad en sus respuestas en cuanto al aspecto de la motivación, "no, la tengo parada, actualmente tengo que retomarla para seguir profundizando en eso".

Siguiendo la entrevista, la informante E respondió: "Si bueno, ahorita por ejemplo yo estoy preparando mi trabajo de ascenso, que tiene que ver con el rol del investigador del docente de la UNESR". Al expresar sobre el impulso interno o fuerza motriz, la mueve el gusto hacia el conocimiento científico: "Yo creo que yo nací para eso, yo soy socióloga, no sé si en la universidad en mi carrera de pregrado me generó esa motivación, o yo nací con eso; pero a mí siempre me ha gustado eso: indagar, buscar, escudriñar y claro ahora con la experiencia, hay un proceso de sistematización, ya uno tiene herramientas, técnicas, herramientas metodológicas para organizar mejor la información".

En esa misma relación el informante $F$ señaló estar activo en la investigación así lo hizo saber: "Sí, yo trabajo el área de investigación". Dentro de sus respuestas dejó ver que disfruta estar investigado: “(..) motiva a investigar el mismo hecho que me motiva la docencia, el ánimo de descubrir cosas nuevas, el ánimo de innovar, obtener conocimientos dentro de mi área específica, conocer en cuanto a didácticas, yo lo disfruto mucho, en cuanto a mis experiencias, cuando yo trabajo así hay muchas cosas nuevas que me abren otras posibilidades de desarrollo".

La última informante codificada con la letra $G$ se le consultó si está realizando investigación y cuál es la motivación, respondió estar activa en el campo de la investigación, así lo manifestó: "Sí, estoy realizando una o varias digámoslo así, de una parten varias, en una soy coordinadora y en otra formo parte como investigadora y en otra estoy como participante". Se observa que las investigaciones de postgrado pueden haber sido un agente motivador; pero el hecho de que ya las terminó y de estar activa demuestra que es investigadora por convicción y lo disfruta: “(...) nos motivó hacer la tesis de las maestrías, yo hice una por la 
Universidad de Carabobo, otra por la Simón Rodríguez, luego el doctorado, siempre hemos estado en constante formación, preparación, la motivación ha sido contagiosa".

El resumen de esta categoría revela la coincidencia de la mayoría de los actores sociales al estar activos investigando; y la motivación hacia el tema de la investigación surge por inquietud o necesidad de los facilitadores, por diagnosticar la situación académica, uniéndose la actualización, la innovación, docencia, obtener conocimientos, satisfacción; sin embargo el análisis lleva al investigador a afirmar que la principal motivación existente en los facilitadores o docentes de la Universidad "en estudio, viene dada a razones académicas personales.

En función de otra categoría se puede decir que los valores o lo axiológico del investigador forman parte de su actitud y es de gran importancia, ya que de alguna manera marcan el rumbo en cuanto al aspecto ético que se debe manejar a la hora de abordar ciertos temas de la vida cotidiana. Según Ruiz (2010) es

la valoración autorreferencial que asumen los investigadores. (...) su espíritu científico, y los valores que en este se constituyen. (...) centra su atención en la forma como el sujeto piensa, actúa, reflexiona. El ser investigativo es una actitud o disposición subjetiva del investigador que busca soluciones serias con métodos adecuados al problema que pretende resolver (...). Puede ser aprendida, pero no es heredada". (p.195)

Con respecto a la conducta ese aspecto observable de la persona marcado por su comportamiento es definida por el autor antes citado como

...el trasluz de donde proceden las normas, los valores, las creencias, el lenguaje, la identidad y el conjunto de los modos de ser, conocer y hacer. (...) el ethos como lo evidente en la conducta social es lo que da forma a la comunidad ya que integra todos sus miembros en un conjunto y conforma una identidad. (Ruiz, 2010, p.197)

Para conocer los valores y la conducta del grupo estudiado, se les preguntó a los entrevistados ipor qué crees que solo un bajo porcentaje de facilitadores se dedican a la Investigación, en la Universidad "Simón Rodríguez" Núcleo San Carlos? La entrevistada A señaló la falta de promoción, así lo dejó entrever respondiendo: "desde mi punto de vista, es un mundo muy cerrado, que los pocos investigadores que realizan trabajos se encuentran ensimismados en ellos. No hay cabida, falta promoción, y entusiasmo, se encuentran desmotivados para poder comenzar a realizar investigación. También depende de parte nuestra poder y querer emprender una investigación".

A la misma pregunta el entrevistado B, afirmó la existencia de muchas opiniones sobre el tema; "quizás hay muchas opiniones diversas; sin embargo, la mayoría o mejor dicho una minoría es la que se mantienen activos en su rol de investigador".

El informante $C$ señaló que la actuación de los facilitadores ha estado determinada en la búsqueda de obtener mejores ingresos económicos y mejorar la calidad de vida, tal como se transcribe: "entonces la universidad es como un complemento a nivel económico, no realmente dedicados a la investigación, (...). La parte económica ha influido demasiado y eso no ha estado 
o no ha sido ausencia de lo que está presentándose en la universidad; por eso es que la investigación, cada vez queda más relegada y aquellos que en su momento o aquellos que han salido de pre-grado y quieran insertarse en una maestría, no es por el área de la investigación, créemelo, es por el área, (...) para que en sus trabajos los puedan ascender".

En ese mismo orden ideas, el informante D indicó que el comportamiento de los facilitadores hacia la investigación, ha estado inspirada en valores o principios tendientes a la búsqueda del incentivo económico a través del programa estímulo al investigador y emprendedor (PEI) y por voluntad propia. “...Hay unos que están realizando investigación por cuenta propia, (...) de esa manera espontánea por su propia voluntad, entonces después ahora surge el PEI, que les ha dado motivo para que otros profesores se sigan incorporándose al proceso de investigación (...) el mismo grupo de profesores son los que trabajan en el área y los que pertenecen a las líneas de investigación no se han incorporado más".

La entrevistada E evidenció la existencia del interés individual hacia la investigación, faltando la unidad de toda la comunidad universitaria, especialmente lo que pudiera evidenciar esto es una política institucional poco motivadora en materia de investigación. Dicha conducta se observa debido a que la universidad exige más en el trabajo administrativo y no hacia la producción investigativa, tal se sustenta: "el que va más allá es porque lo hace por su propia voluntad, no porque la universidad desde el punto de vista institucional como empleador te motiva y te exige más de eso, y el que decide ir más allá como yo, o como otros es por su propia voluntad, entonces yo creo que uno se va adaptando al requerimiento institucional, si la universidad no te exige para que te vas a poner, cuando al profesor le van a dar el cambio de dedicación a tiempo completo es porque la van a dar una coordinación que no necesariamente está vinculada con la investigación es eso, la universidad exige más desde el punto de vista administrativo".

El informante F reveló el protagonismo del paradigma del desinterés por la investigación: "vemos la investigación parcelada de la docencia, la universidad contrata a un docente para dar clase así textualmente y ese docente se va a dedicar a cumplir su labor única y exclusivamente a la docencia, divorciada, (...) pienso que hay un interés personal en el momento en que se aborda la investigación dentro de la universidad, el profesor universitario aborda la investigación solamente con un fin particular y el fin particular es que necesita un trabajo de investigación".

Continuando la narración la informante $G$ respondió: “...son docentes por otra razón económica más no por vocación, para ser interacción, tienes que estar enamorado de un proyecto, de una idea". Las consideraciones de los informantes, muestran una serie de valores que sin duda marcan el camino de la conducta del facilitador, entre ellas ven la investigación como un estilo de vida y con sentimiento cargado de amor hacia los proyectos científicos. Sin embargo, se logró evidenciar otros valores negativos del ser investigativo entre ellos, la poca actitud, escaso espíritu científico, convirtiéndose en una comunidad académica científica cerrada.

En consecuencia, prevalece una cultura del individualismo que separa la investigación de la función docente, donde el facilitador no comprende cuáles son las funciones de un facilitador universitario, ocasionando un incipiente desarrollo investigativo por ausencia de una dirección que motive a investigar con estrategias de fortalecimiento docente, a fin de minimizar 
la conducta del facilitador movido por el interés académico personal.

\section{CONCLUSIONES}

De acuerdo a la interacción entre los informantes claves a través del aporte de las entrevistas y a partir de la situación observada, se dio respuesta a los objetivos de la investigación en cuanto a la actitud hacia la función de investigación de los facilitadores de la Universidad Nacional Experimental "Simón Rodríguez", Núcleo San Carlos, estado Cojedes.

Con respecto al propósito orientado a develar los afectos, cogniciones y la conducta de los facilitadores hacia la función de investigación surge la siguiente comprensión: Se pudo detectar en primer lugar que son pocos los facilitadores activos en la función de investigación. Entre las razones se sustenta la desmotivación o falta de interés. Cabe destacar que existen los facilitadores altamente motivados quienes investigan por el placer que les produce, como una de sus funciones, porque les gusta experimentar cosas nuevas, para obtener conocimientos y para satisfacción personal.

En ese mismo orden de ideas, se apreciaron elementos que influyen negativamente no solo en la función hacia la investigación, sino además la ocupación docente, por la existencia de debilidad en los valores, pues resalta en los facilitadores el interés individual, pues los que investiga lo hacen por cuenta propia y movido por intereses académicos personales. Se une la apatía, falta de compromiso, falta de unidad de la comunidad científica y de identidad hacia la universidad. Por otra parte el escaso espíritu científico debido a que los facilitadores no asisten a cada una de las actividades relacionadas a investigación y a los programa de formación de tutores e investigadores. Sin embargo, existe un grupo de facilitadores que tienen claridad en sus funciones docentes y también amor a la investigación y se preocupan por analizar y buscar soluciones a las distintas problemáticas sociales como el hecho de hacer propuestas para minimizar hechos violentos y fortalecer valores desde la educación.

De allí que se pudo observar un comportamiento de una comunidad académica cerrada con incipiente desarrollo investigativo, en parte por falta de una dirección o gerencia que obligue a investigar y a un cuerpo de facilitadores, el cual aún no visualiza la investigación como parte de la función docente donde priva el interés individual. Aun así un pequeño grupo reconoce los aportes importantes de la investigación.

Con respecto al propósito destinado a describir las funciones de los facilitadores en cuanto a su rol de investigadores, se pudo apreciar una limitante que impide la producción de nuevos conocimientos. En primera instancia una condición laboral limitante de la planta profesoral, el desconocimiento de la función docente, quienes ven de manera separada las tres funciones del facilitador incumpliendo la normativa o leyes que regulan la función docente universitaria. En definitiva, existe un problema estructural ya que los que ejercen la función investigativa, la realizan solo por razones académicas personales, existiendo un interés individual como es mejorar en el escalafón laboral y lograr buenos ingresos económicos. Aun así un pequeño grupo le da importancia por la actualización metodológica y obtener nuevos conocimientos.

Al verificar el aspecto de las líneas de investigación, la indagación arroja que son 
pocos los facilitadores o docentes adscritos a las líneas de investigación creadas de alguna manera por la condición laboral limitante o falta de tiempo (contratados a tiempo convencional), desconocimiento de líneas de investigación traduciéndose en una debilidad organizacional de la universidad en estudio.

Otro aspecto importante de la comprensión se refirió a conocer los programas de formación académica institucional para la investigación que ofrece la Universidad a sus facilitadores, destacándose en los actores sociales, la carencia de formación en investigación aun cuando el núcleo universitario estudiado realiza algunos talleres en temas científicos y de actualidad el apoyo es insuficiente a la investigación y programas. En otras palabras, existe insuficiente formación científica por falta de promoción y motivación hacia la investigación en facilitadores y estudiantes, aunado al desconocimiento de las políticas institucionales investigativas.

Lo antes señalado revela una gran debilidad institucional, pues los facilitadores adscritos al Núcleo de San Carlos, desconocen la existencia de la Dirección de Desarrollo Profesoral de la que depende la división de actualización y desarrollo y en los Núcleos las coordinaciones de investigación y el Consejo de Desarrollo Científico, Humanístico, Tecnológico (CDCHT) y a nivel del núcleo la desaparición de diplomados. La inversión financiera $y$ presupuestaria dedicada a los programas de formación e investigación es deficitaria otorgándosele poca importancia a la investigación. Por otra parte, la divulgación y comunicación científica es nula, al no existir promoción de resultados de los trabajo científicos, ninguna revista científica y ni laboratorios.

En suma, existe en el núcleo de esta universidad una debilidad por la contratación de facilitadores en su mayoría graduados en carreras diferentes a la docencia, por directrices emanadas de nivel central que no se ajustan a la realidad, afectando al hecho educativo, ya que no son docentes y si fueran docentes se sentirían investigadores por naturaleza. Son pocos los docentes que desarrollan sus funciones en investigación, escasos los adscritos a líneas de investigación donde existen proyectos por los cuales los facilitadores no muestran interés. Por lo tanto, la universidad, debe fortalecer las políticas institucionales hacia la función docente investigativa a través del estímulo con buena asignación presupuestaria y de inversión para darle más estímulo a la investigación.

Finalmente, se concretaron los hallazgos de cada uno de los propósitos desde las distintas categorías estudiadas, existiendo coincidencia entre lo observado (diario de campo) y en las entrevistas. Parte de los descubrimientos lleva a afinar en cuanto a la situación problemática, es que hay fallas en la implementación de las políticas, en el seguimiento y control de los proyectos y en el poco seguimiento al cumplimiento de las funciones docentes.

REFERENCIAS

Aldana, G. M., y Joya, N. S. (2011). Actitudes hacia la investigación científica en docentes de metodología de la investigación. Tabula Rasa, (14), 295-309

Arias, F. (2006). El Proceso de Investigación. (4르 ed) Caracas: Episteme

Boffelli, P. (2015). Enseñanza de la investigación desde la perspectiva de la investigación acción. (Tesis Doctoral no publicada) Universidad Nacional Experimental. 
Simón Rodríguez, Núcleo Barquisimeto

Castillo, E., y Vásquez, M. L. (2003). El rigor metodológico en la investigación cualitativa. Colombia médica, 34(3), 164167

Consejo Superior de Investigaciones Científicas (CSIC) (2012). Recuperado de

http://www.leanoticias.com/2012/03/05/ ranking-de-universidades-en-venezuela/

Heidegger, M. (2003). Ser y Tiempo. Madrid España: Trota

Hurtado. J. (2000). Metodología de la investigación holística. (3르 ed). Caracas: Sypal

Ley de Universidades (1970). (Gaceta oficial № 1429). Recuperado de http://www.ucv.ve/fileadmin/user_uploa d/auditoria_interna/Archivos/Material_d e_Descarga/Ley_de_Universidades__. 1.429 E.pdf

Martínez, M. (2002). Comportamiento humano: Nuevos métodos de investigación. ( $2^{\underline{a}}$ ed). México: Trillas

Martínez, M. (2006). Validez y confiabilidad en la metodología cualitativa. Paradigma N.2. p. 07-33
Miguélez, M. M. (2006). Fundamentación epistemológica del enfoque centrado en la persona. Polis, Revista Latinoamericana, 5(15)

Martínez, A. (2008). El Docente Universitario y su Espacio de Formación, Fundamentación de una Propuesta. Revista Docencia Universitaria, Universidad Central de Venezuela, Vol. IX, (№ 2.), 55

Reglamento General de Educación Avanzada UNESR (2011). Universidad Nacional Experimental "Simón Rodríguez". Rectorado-Publicaciones del Decanato de Postgrado

Ruiz, G. (2010). La investigación como cultura relegada en la facultad de ciencias de la educación, Universidad de Carabobo. Revista de Postgrado FaceUC Arjé 4 (7), 35-39

Sánchez, J. (2015). Formación docente e investigación en el contexto de la educación venezolana actual. Recuperado de http://www.saber.ula.ve/bitstream/1234 56789/16539/1/ponencia8.pdf 